\title{
Reccurent Sebaceous Gland Carcinoma of the Eyelid with Metastasis to the Parotid Gland and the Regional Lymph Nodes: Case Report and Review of the Literature
}

\author{
Vafeiadou Margarita ${ }^{1 *}$, Alexoudi Vaia-Aikaterini ${ }^{1}$, Kilmpasani Maria ${ }^{2}$, Karafoulidou loanna ${ }^{2}$ and \\ Antoniades Konstantinos ${ }^{1}$ \\ ${ }^{1}$ Department of Oral and Maxillofacial Surgery, Aristotle University of Thessaloniki, Greece \\ ${ }^{2}$ Department of Pathology, General Hospital of Thessaloniki "G. Papanicolaou", Thessaloniki, Greece
}

\begin{abstract}
Sebaceous cell carcinoma is a rare cutaneous aggressive tumor arising from the sebaceous glands. . Its almost exclusive site of occurrence is the eyelid but it has also been reported primarily in other sites, such as the parotid region. It is one of the most dangerous tumors, due to masquerading as inflammatory conditions, such as blepharoconjunctivitis, superior limbic keratoconjunctivitis or chalazion. It may also be misdiagnosed as other malignancies, while the incidence of metastasis is high-about $41 \%$.

In our article we report a rare case of a reccurent sebaceous gland carcinoma of the eyelid with metastasis to the parotid gland and the regional lymph nodes.

In the review of the literature, diagnosis, treatment and the prognosis of this rare entity are described.
\end{abstract}

\section{Introduction}

Sebaceous cell carcinoma is a cutaneous aggressive tumor arising from the sebaceous glands. It is considered as the third most common eyelid malignancy after basal and squamous cell carcinomas. Its almost exclusive site of occurrence is the eyelid but it has also been reported primarily in other sites, such as the parotid region. Among western population it accounts for $<1-5.5 \%$, while in Asians it can be observed at about $31-39 \%$ [1-7].

Sebaceous cells are predominantly present in meibomian glands of the tarsal plate, sebaceous glands of ocular adnexa (gland of Zeis), sebaceous glands embedded in caruncle, brow and associated hair follicles of lid skin. The most common site of occurrence is the upper eyelid.

It has been reported as the great masquerader, due to its resemblance to other benign or malignant lesions. In the differential diagnosis chalazion, blepharitis, conjunctivitis, basal cell carcinoma, squamous cell carcinoma, leukoplakia, ocular pemphigoid and carcinoma in-situ must be concerned [1,5,8-10].

We report a case of a parotid metastasis originating from a carcinoma of the upper eyelid, diagnosed as sebaceous cell carcinoma. Diagnosis and treatment of this entity are described.

\section{Case Presentation}

An 80-year-old Caucasian woman presented in our clinic with a firm, painless, slowly enlarging mass at her right parotid area with normal overlying skin. The patient had no regional palpable neck nodes. She also presented with a diffused eyelid, thickening in the upper right eyelid, painful in pulpation.

In her medical history she mentioned type I diabetes under medication. The patient had no history of tobacco or alcohol use. In her surgical history, she mentioned excision of a mass of the right lower eyelid four years ago, diagnosed as a squamous cell carcinoma of the skin of the eyelid, and which had been excised in whole, with histologically free margins of excision and depth invasion $4 \mathrm{~mm}$. The patient had taken

*Corresponding author: Vafeiadou Margarita, Department of Oral and Maxillofacial Surgery, Aristotle University of ThessaIoniki, Thessaloniki 541 24, Greece

Accepted: June 08, 2019

Published online: June 10, 2019

Citation: Margarita V, Vaia-Aikaterini A, Maria K, et al. (2019) Reccurent Sebaceous Gland Carcinoma of the Eyelid with Metastasis to the Parotid Gland and the Regional Lymph Nodes: Case Report and Review of the Literature. J Head Neck Surg 2(1):13-17

Copyright: (c) 2019 Margarita V, et al. This is an open-access article distributed under the terms of the Creative Commons Attribution License, which permits unrestricted use, distribution, and reproduction in any medium, provided the original author and source are credited. 
Citation: Margarita V, Vaia-Aikaterini A, Maria K, et al. (2019) Reccurent Sebaceous Gland Carcinoma of the Eyelid with Metastasis to the Parotid Gland and the Regional Lymph Nodes: Case Report and Review of the Literature. J Head Neck Surg 2(1):13-17

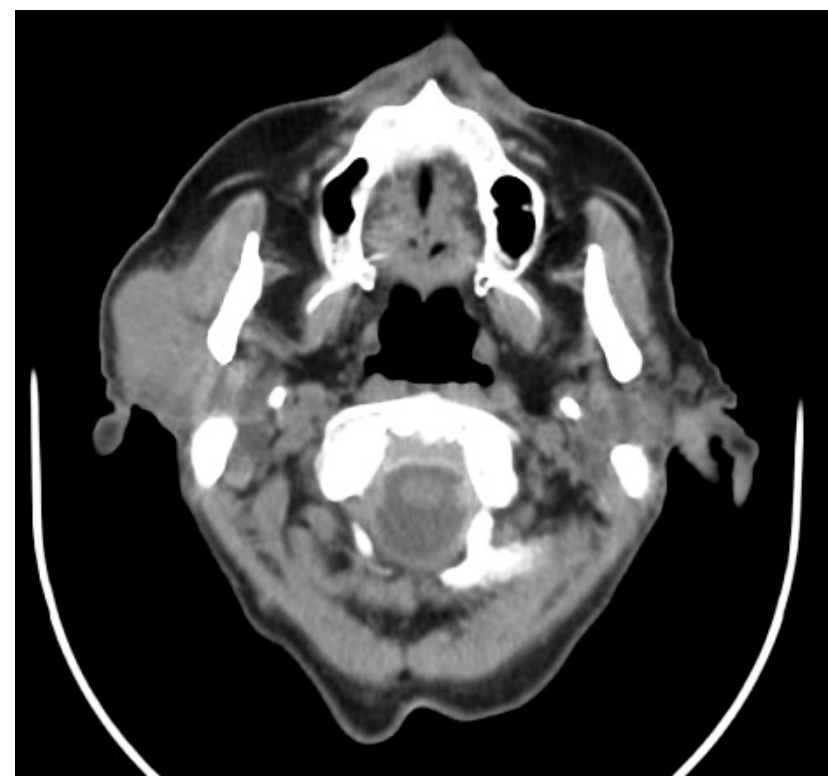

Figure 1: CT scan indicating a compact mass in the right parotid gland, with dimensions about $36 \times 20 \times 30 \mathrm{~mm}$.

no supplementary treatment for that tumor. Due to that history, the mass of the right parotid was considered to be a metastasis from the SCC of the eyelid.

The CT scan (Figure 1) and MRI of head and neck (Figure $2 \mathrm{a}$ and Figure $2 \mathrm{~b}$ ) indicated a compact mass in the right parotid gland, with dimensions about $36 \times 20 \mathrm{~mm}$. The regional lymph nodes were depicted normal.

Full staging was performed preoperatively, including radiographic control of the chest and ultrasound of the upper abdomen that revealed no distant metastasis.

Ophthalmic examination was also performed. Her visual acuity in the right eye was $1 / 10$ in the central position and $2 / 10$ peripherally, without diplopia, proptosis, and displacement of eye ball or limitation of extra-ocular movements. There was also extended conjunctival filtration. The left eye suffered from an operable senile nuclear cataract.

Under general anesthesia, a supraomohyoid selective neck dissection was performed, followed by a radical parotidectomy with sacrifice of the facial nerve and exenteration of the right eye ball. The periorbital muscles were left intact for potential prosthetic reconstruction and the deficit was closured with regional flaps.

The histopathologic evaluation of the lesions revised the previous diagnosis of a squamous cell carcinoma and stated a diagnosis of a sebaceous gland carcinoma. This infiltrated the upper and the lower eyelid, either in situ (Figure 3a) or invading the underlying stroma, showing well and poorly differentiated areas (Figure $3 \mathrm{~b}$ ) and demonstrated a pagetoid spread to the bulbar conjunctiva (Figure 3c). The excision was undertaken in histologically healthy margins. The lesion in the parotid gland proved to be a metastasis of the sebaceous gland carcinoma, demonstrating necrotic areas (Figure 3d). An intraparotid lymph node, as well as a lymph node of level II in the neck dissection, among the total of 10 lymph nodes
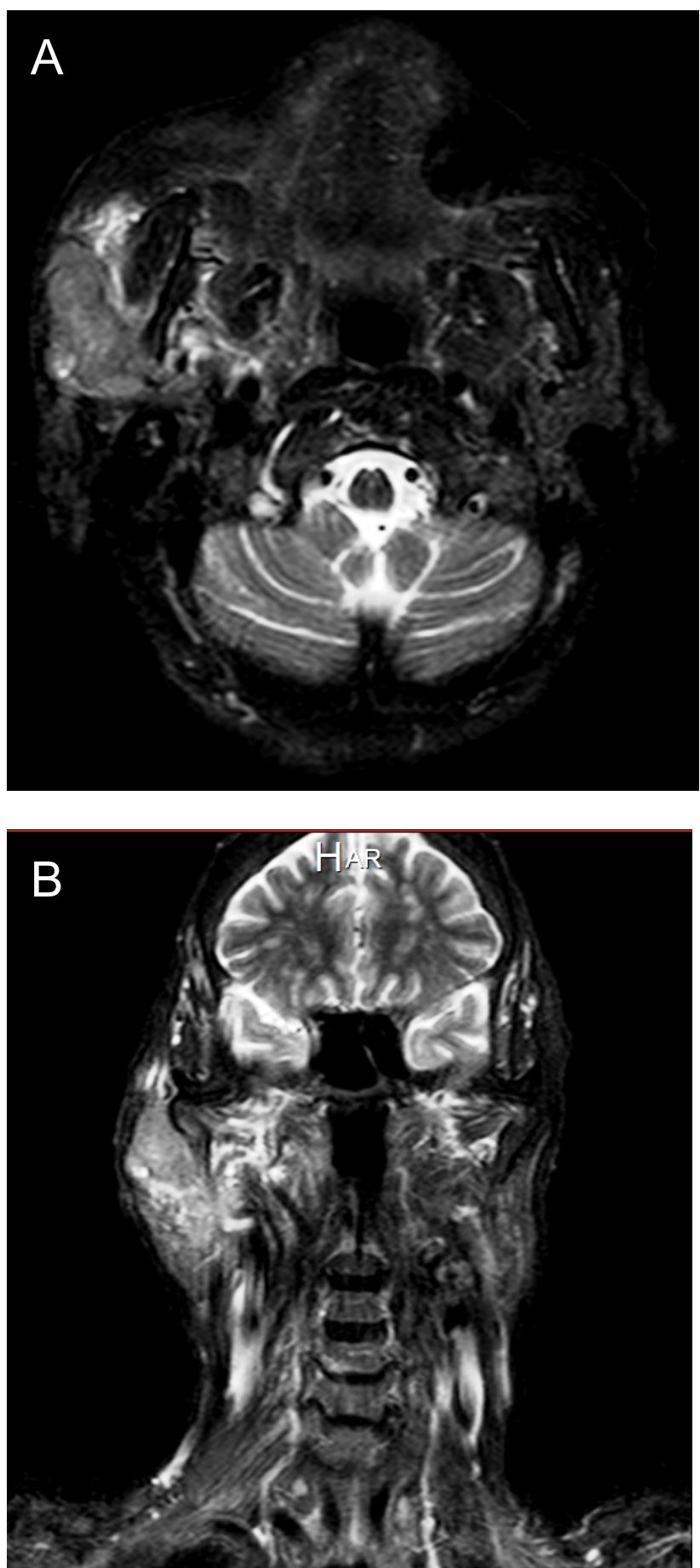

Figure 2: a,b) MRI T2 sequence: the same mass with dimensions $36 \times 20 \times 30 \mathrm{~mm}$ with heterogeneous slightly increased sign.

that had been dissected, were positive for metastasis of the above carcinoma.

The patient underwent further radiotherapy and is under regular follow-up in the external unit of our clinic (Figure 4a, Figure $4 b)$. There is no recurrence so far.

\section{Discussion}

Sebaceous carcinoma, sebaceous gland carcinoma (SGC), sebaceous cell carcinoma and meibomian gland carcinoma are all terms used in the literature to malignant neoplasm of 
Citation: Margarita V, Vaia-Aikaterini A, Maria K, et al. (2019) Reccurent Sebaceous Gland Carcinoma of the Eyelid with Metastasis to the Parotid Gland and the Regional Lymph Nodes: Case Report and Review of the Literature. J Head Neck Surg 2(1):13-17
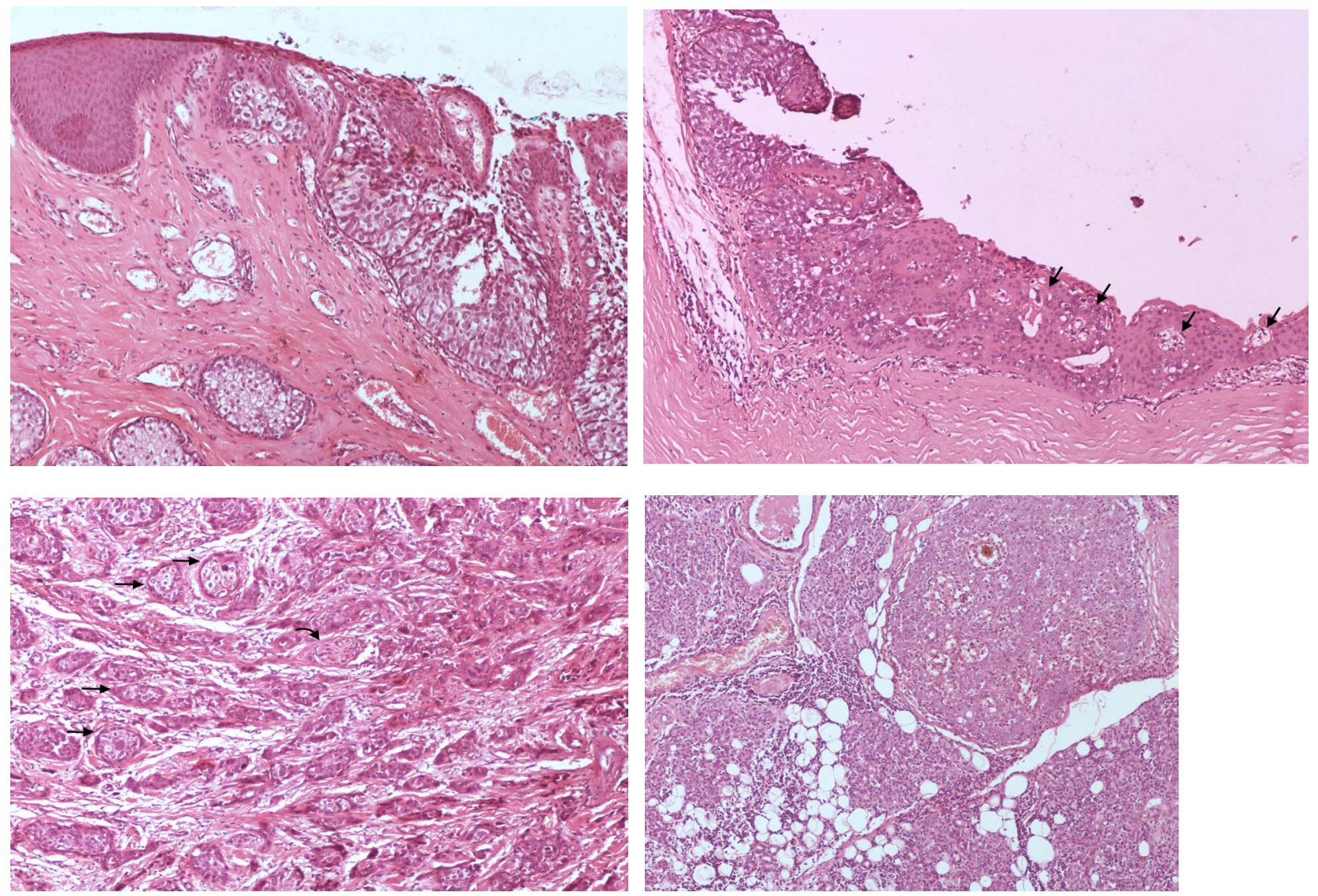

Figure 3: a) Conjuctival epithelium infiltrated by sebaceous gland carcinoma in situ. The neoplastic cells demonstrate sebaceous differentiation and a papillary growth pattern in this area. Uninvolved normal sebaceous glands are seen in the lower left corner. ( $\times 100)$; $b$ ) The carcinoma replaces the epithelium of the palpebral conjuctiva, exhibiting a papillary growth pattern (upper left corner), and extends to the conjuctival cul de sac and the bulbar conjuctiva (bottom) in a pagetoid fashion (arrows). (x100); c) In the well differentiated area (left) the neoplastic cells have vacuolated cytoplasm and form lobules, showing evident sebaceous differentiation (arrows), while the poorly differentiated area (right) consists of cells with variable size, more eosinophilic cytoplasm and prominent nuclear pleomorphism. A perineural invasion can be seen in the center (hooked arrow). (x100); d) Effacement of the architecture of the parotid gland due to metastasis of the carcinoma (upper right corner) $(\times 100)$.

sebaceous origin. Sebaceous carcinoma is traditionally classified into two groups: Tumor arising from the ocular adnexa in the periocular area and those arising in the extraocular sites, which commonly involve the head and neck region, the parotid and submandibular glands, the external auditory canal, the trunk and laryngeal or pharyngeal cavities. It is considered to be the third most common eyelid malignancy - after basal cell and squamous cell carcinoma - representing 1-5.5\% of all eyelid tumors. However, it is one of the most dangerous tumors, due to masquerading as inflammatory conditions, such as blepharoconjunctivitis, superior limbic keratoconjunctivitis or chalazion. It may also be misdiagnosed as other malignancies. The result is delayed diagnosis or wrong treatment [1,3,5,7-11].

In addition to its variable clinical appearance, a variable histologic appearance may also occur. As a result, delayed diagnosis or misdiagnosis following biopsy is not uncommon. Eyelid sebaceous gland carcinoma has a characteristic intraepithelial growth pattern, resulting from a pagetoid spread or a full-thickness replacement of the surface epithelium. The tumor may demonstrate well, moderately or poorly differentiated areas. The histologic examination may reveal a papillary or lobular growth pattern of neoplastic cells with evident sebaceous differentiation, or highly atypical neoplastic cells with prominent nuclear pleomorphism. Areas of necrosis and fibrosis are commonly found. The spread of the tumor in the form of infiltrating lobules, nests and cords is characteristic, perineural invasion is observed in about $20 \%$ of tumors, whereas vascular invasion is extremely infrequent $[2,4,12-14]$.

The treatment of SGCs is adequate surgical excision with wide surgical margins and fresh frozen section controls. When the tumor diffuses the eyelid, orbital exenteration is required. Radical or selective neck dissection should be performed, to evaluate lymph node metastasis. Adjunctive radiotherapy or chemotherapy are necessary, depending on the stage of the tumor at the time of presentation, or when the surgical margins are diffused $[1,3,6,8,9,11,13,15,16]$.

In our case, the patient presented with a parotid gland metastasis. Given her history of a surgical excision of a squamous cell carcinoma of the lower eyelid 4 years ago, the parotid gland tumor was thought to be a metastatic SCC. Unfortunately, we had no chance to histologically reexamine the 
Citation: Margarita V, Vaia-Aikaterini A, Maria K, et al. (2019) Reccurent Sebaceous Gland Carcinoma of the Eyelid with Metastasis to the Parotid Gland and the Regional Lymph Nodes: Case Report and Review of the Literature. J Head Neck Surg 2(1):13-17
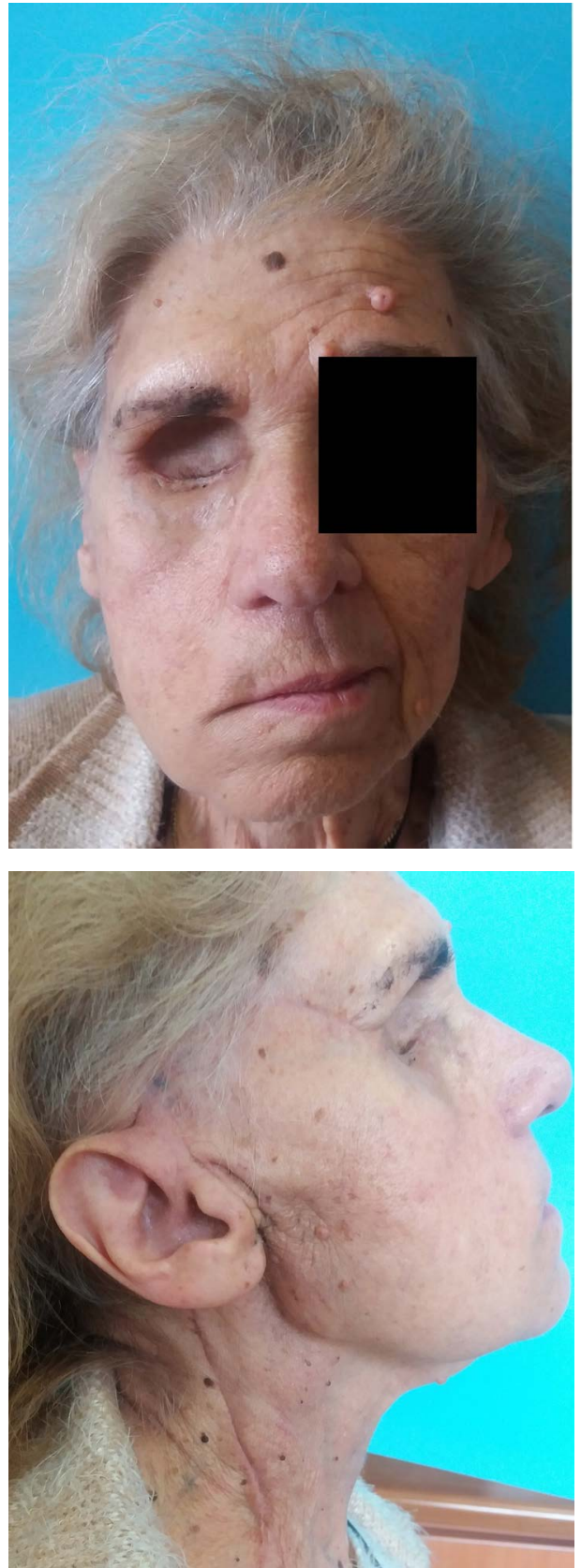

Figure 4: a,b) The patient postoperatively, after the complication of the radiotherapy.

previous tumor, in order to clarify the incidence of misdiagnosis. In the histological examination of the present tumor in the eyelids, the characteristic features of a sebaceous gland carcinoma were observed. Furthermore, the tumor in the parotid gland demonstrated the same features, and was proven to be a metastasis of the above carcinoma, while metastases in two regional lymph nodes were also noticed. In the literature, there are only 3 cases reported with metastasis to the parotid gland, while the tumor most commonly metastasizes to the lymph nodes.

SGCs have a high incidence of recurrence. Recurrence is a major problem and can be at the same site or at a different location. It is difficult to say whether it is a true recurrence or instead a new tumor, due to the multicentric nature of SCG.
Well-differentiated tumors have a better prognostic outcome in compare to the poorly differentiated. Confirmation of pagetoid spread is another bad prognostic factor. Other adverse prognostic features include the involvement of both upper and lower eyelid, tumor size more than $10 \mathrm{~mm}$, orbital extension and infiltration of blood vessels and/or lympatics $[1,3,5-7,10]$.

Metastasis can occur by continuous growth, lympatic spread or hematogenous spread. The most common sites of continuous growth is the orbit, the periaucular area and the parotid gland, as well as the regional lymph nodes of level I and II. More rare is distant metastasis to the lung, pleura, liver, brain, pericardium, lips, ethmoid sinus and skull. The overall mortality rate is $5-10 \%$, while in cases of metastasis the rate may go up to $25 \%$ and up to $41 \%$ in cases where the duration of symptoms lasts more than six months $[1,3,8,9,11$ 14].

\section{Conflict of Interest Declaration}

The authors declare no conflicts of interest regarding the publication of this paper.

\section{References}

1. Rizvi SA, Alam S, Akhtar K (2018) Eyelid sebaceous gland carcinoma: Varied presentations and reconstruction outcome. Oman J Ophthalmol 11: 21-27.

2. Kanwalpreet Kaur, Karuna Gupta, Kalpana Mangal, et al. (2017) Histopathological Analysis of Skin Adnexal Tumors: A Three Year Study of 110 Cases at A Tertiary Care Center. Indian J Dermatol 62: 400-406.

3. El Amin Marnouche, Abdelhak Maghous, Noureddine Benjaafar, et al. (2016) Sebaceous carcinoma of the parotid gland: a case report and review of the literature. J Med Case Rep 10: 174.

4. Prem Chand, Ashok Kumar, Lakshay Singla, et al. (2016) Unusual Presentation of Ulcerative Postauricular Swelling as Sebaceous Cell Carcinoma. Niger J Surg 22: 127-129.

5. Upender K Wali, Abdullah Al-Mujaini (2010) Sebaceous gland carcinoma of the eyelid. Oman J Ophthalmol 3: 117-121.

6. Evan Silverstein, Marcus Marcet (2019) Sebaceous carcinoma. American Academy of Ophthalmology.

7. Glassman M, Roy H (2017) Sebaceous Gland Carcinoma. Medascape.

8. Shields JA, Demirci H, Marr BP, et al. (2004) Sebaceous carcinoma of the eyelids: Personal experience with 60 cases. Ophthalmology 111: 2151-2157.

9. Waseem M, Humayun S (2010) Ayaz B Sebaceous Carcinoma of Upper Eyelid Journal of the College of Physicians and Surgeons Pakistan 20: 487-489.

10. Fan B, Liu JJ, Li GY, et al. (2017) Case report repairing orbital skin defects using composite flaps after giant eyelid-derived tumor excision and orbital exenteration. Medicine Baltimore 96: e8978.

11. Song A, Carter KD, Syed NA, et al. (2008) Sebaceous cell carcinoma of the ocular adnexa: clinical presentations, histopathology, and outcomes. Ophthalmic Plast Reconstr Surg 24: 194-200.

12. Hashimoto K, Yasumatsu R, Toh S, et al. (2016) Patterns of lymphatic spread and the management of eyelid carcinomas. Auris Nasus Larynx 43: 666-671. 
Citation: Margarita V, Vaia-Aikaterini A, Maria K, et al. (2019) Reccurent Sebaceous Gland Carcinoma of the Eyelid with Metastasis to the Parotid Gland and the Regional Lymph Nodes: Case Report and Review of the Literature. J Head Neck Surg 2(1):13-17

13. Soares CD , Morais TML, Carlos R, et al. (2018) Sebaceous adenocarcinomas of the major salivary glands: a clinicopathological analysis of 10 cases. Histopathology 73: 585-592.

14. SV Deo, Nootan Kumar Shukla, Mandeep Singh, et al. (2012) Locally Advanced Sebaceous Cell Carcinoma (T3) of Eyelid: Incidence and Pattern of Nodal Metastases and Combined Modality Management Approach. Orbit 31: 150-154.
15. Lally SA, Rao R, Sheilds JA, et al. (2018) Comparison of posterior lamellar resection versus lumpectomy for initial management of localized tarsal conjunctival sebaceous carcinoma in 54 cases. Indian J Ophthalmol 66: 1295-1300.

16. Sandosh G Honavar (2018) Sebaceous gland carcinoma: Can we do better? Indian J Ophthalmol 66: 1235-1237.

DOI: $10.36959 / 605 / 530$

Copyright: (c) 2019 Margarita V, et al. This is an open-access article distributed under the terms of the Creative Commons Attribution License, which permits unrestricted use, distribution, and reproduction in any medium, provided the original author and source are credited. 